\title{
Components of displacement adaptation in acquisition and decay as a function of hand and hall exposure
}

\author{
GORDON M. REDDING \\ Illinois State University, Normal, Illinois 61761 \\ and \\ BENJAMIN WALLACE \\ Western Illinois University, Macomb, Illinois 61455
}

\begin{abstract}
Tests of proprioceptive shift (PS), visual shift (VS), and negative aftereffect (NA) were made during 25-min exposure to 20-D displacement and during a subsequent 30 -min dark decay period in two separate experiments. Different groups of subjects explored hallways or viewed their active hand during exposure. VS was greatest in hall exposure, while PS was greatest in hand exposure. Larger VS occurred in the second experiment, where test procedures were modified to minimize a tendency to center the target within the momentary or remembered field of view. Substantial and possibly complete VS decay occurred when the initial level of adaptation was high, but although PS decay was substantial, it was not complete. In all conditions, the sum of VS and PS numerically exceeded the NA, and this difference tended to be largest and significant in the hall exposure. Implications of this effect for the two-component additivity hypothesis are discussed.
\end{abstract}

Much of the current emphasis in research on perceptual adaptation is concerned with identifying various kinds of adaptive change and with stating the rule governing how the various components combine to produce total adaptation. Since the conditions for adaptation appear to involve the entire control loop that regulates our interaction with the world, it is unlikely that adaptive change will be restricted to one part of this system. More likely, multiple changes occur in various parts of the system. There is considerable evidence (e.g., Hay \& Pick, 1966; McLaughlin \& Webster, 1967) for adaptive change in at least two parts of the control loop: those responsible for visual appearance and for felt-limb position. Wilkinson (1971) has proposed that displacement adaptation can be accounted for by two such components: change in visual direction and in felt-hand position. Moreover, he suggested a simple linear model for the combination of components. Changes in eye-hand coordination, which is presumably sensitive to both kinds of adaptation,

A partial and preliminary report of this study was read at meetings of the Midwestern Psychological Association, Chicago, Illinois, May 1976. The research was supported in part by a research grant from Illinois State University to Gordon $\mathbf{M}$. Redding, and a grant from the Western Illinois University Research Council to Benjamin Wallace. The authors also wish to thank Linda Edwards and Edward Mayse for their assistance in data collection. Reprint requests should be addressed to Gordon M. Redding, Department of Psychology, Illinois State University, Normal, Illinois 61761 . should be the linear sum of proprioceptive and visual components. Wilkinson reported data supporting this two-component model and Wallace (Note 1; Wallace \& Garrett, 1975) also found evidence for such additive effects. However, Welch, Choe, and Heinrich (1974) failed to find additivity, and suggested that an additional component might be necessary to account entirely for adaptive changes in eyehand coordination. Also, Templeton, Howard, and Wilkinson (1974) failed to find two-component additivity under certain conditions that suggest the operation of sensorimotor coordinators (Hardt, Held, \& Steinbach, 1971) between parts of the control loop system.

The research reported here is based on the premise that a true test of the linear model depends upon the ability to manipulate the relative magnitude of adaptive components. Previous studies have made no attempt to manipulate components, being content to construct tests designed to differentially measure the various components. One reason for the ambiguous state of the model may be this lack of direct control over the mediating processes.

Redding (Note 2) found exposure conditions that appear to differentially affect visual and proprioceptive components of tilt adaptation. Proprioceptive change was greater when subjects viewed the active hand during exposure, while visual change was greater when exposure consisted of hallway exploration. Presumably, the absence of any direct conflict between vision and proprioception and the free 
exploration in hall exposure maximizes visual adaptation, while the restricted viewing conditions of hand exposure maximizes proprioceptive adaptation. Similar reasoning can be applied to displacement adaptation, and the present study attempted to control components of adaptation to displacing prisms by having different groups of subjects in hand and hall exposure conditions. Also in the present study, visual and proprioceptive components of displacement adaptation were measured repeatedly both during acquisition and during a subsequent dark decay period. This procedure provides a strong test of the two-component model since additivity is required at different levels of adaptation.

Another question of interest in this study was concerned with the time course of decay. Redding (1975) found data suggesting that decay of the visual component of displacement adaptation is complete, reaching zero after a dark period approximately equal in length to the exposure period. Choe and Welch (1974), however, found essentially no decay. of visual adaptation during a dark period which was much longer than the initial exposure. One of the differences between these two studies which might account for the different decay functions was exposure conditions. Choe and Welch had their subjects view their active hands during exposure, while Redding's subjects explored hallways. The present study, therefore, provided for assessment of decay following both hand and hall exposure.

\section{EXPERIMENT 1}

\section{Method}

Subjects. The subjects were undergraduate volunteers at Western Illinois University. The $\mathbf{3 6}$ male and female volunteers received credit toward course performance for their participation.

Procedure. During hall exposure, subjects walked about hallways wearing goggles with a Risley prism mounted over the right eye which displaced the visual field $20 \mathrm{D}$ in the rightward direction. The subjects were instructed not to look at their hands or feet, and the narrow field of view (approximately $20^{\circ}$ ) virtually precluded sight of any part of the body. During hand exposure, subjects watched one of their hands, with a slide attached to forefinger and mounted on a track in a Held and Gottlieb (1958) type of apparatus (illustrated in Melamed, Halay, \& Gildow, 1973), while they moved the arm in a lateral motion within the visual field. A metronome timed this back-and-forth slide movement, each cycle requiring $6.0 \mathrm{sec}$ for completion. The left eye was occluded throughout the experiment, and experimental sessions required a total time of approximately $1.5 \mathrm{~h}$.

To test for visual shift (VS), the subjects adjusted a target moved laterally in the visual field to appear straight ahead of the nose. Adjustments were made by verbal instructions to the experimenter, who actually moved the target. The proprioceptive shift (PS) test required the subjects to laterally adjust a nonvisible slide with the index finger to feel straight ahead of the nose. The negative aftereffect (NA) test required coordination of the eye and hand in a task presumed sensitive to both visual and proprioceptive adaptation. The subjects laterally adjusted a nonvisible slide with the index finger to alignment with a visible straight-ahead target. ${ }^{1}$ All tests were conducted monocularly, without prisms, in a lighted field which was homogeneous except for the necessary test targets. Each of the three tests was given before and at 5 -min intervals during a total exposure of $25 \mathrm{~min}$ and during a subsequent dark decay period of $30 \mathrm{~min}$ (five exposure and six decay tests).

Design. Subjects were alternately assigned to exposure conditions, 18 in the hall and 18 in the hand condition, as they appeared for experimentation. Order of the three tests was completely counterbalanced within groups, 3 subjects in each group receiving each of the six test orders. Baseline performance prior to exposure was measured for each of the three tests, and level of adaptation (LA) was defined as the difference between pre- and postexposure performance. Positive signs were given to performance changes in the rightward (adaptive) direction on the VS test, and to changes in the leftward (adaptive) direction on the PS and NA tests.

\section{Results and Discussion}

Figure 1 shows LA as a function of exposure and decay time for each of the three kinds of tests. The last acquisition test at $25 \mathrm{~min}$ is also the first decay test at $0 \mathrm{~min}$. The top portion of the figure represents performance under hand exposure, and the bottom portion shows the results for hall exposure. Separate analyses of variance were performed for acquisition and decay data.

Acquisition. In acquisition, overall adaptation $\left(1.26^{\circ}\right)$ was significantly greater than zero, $F(1,34)=$ $68.24, p<.001$, and adaptation generally increased with increasing exposure time, $F(4,136)=27.96$, $\mathrm{p}<.001$. Significant main effects also appeared for the kind of test, $F(2,68)=3.94, p<.025$, and for

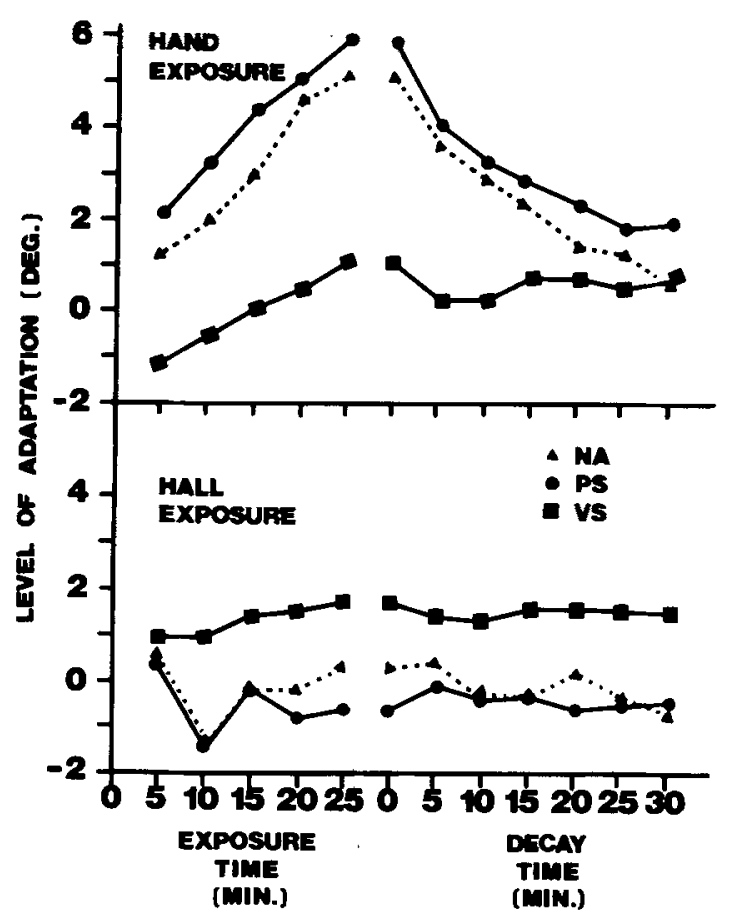

Figure 1. Level of adaptation as a function of exposure and decay time for hall and hand exposure conditions as measured by three kinds of tests: negative aftereffect (NA), proprioceptive shift (PS), and visual shift (VS). The positive direction indicates adaptive change for each test. Experiment 1. 
groups, $F(1,34)=48.86, p<.001$, with the handexposure group $\left(2.33^{\circ}\right)$ showing greater adaptation than the hall-exposure group $\left(0.19^{\circ}\right)$. Moreover, the interaction of kind of test and exposure groups was significant, $F(2,68)=30.05, p<.001$, indicating that the hand vs. hall exposure manipulation was successful in affecting the relative magnitude of PS and VS. With hand exposure, the average PS $\left(4.02^{\circ}\right)$ was greater than the average VS $\left(-.03^{\circ}\right)$. Conversely, with hall exposure, the average VS $\left(1.32^{\circ}\right)$ was greater than the average PS $\left(-.54^{\circ}\right)$. The only other significant source of variance in acquisition was the Test by Groups by Time interaction, $F(8,272)=3.25$, $\mathrm{p}<.005$. Examination of Figure 1 shows that PS and NA both increased during hand exposure, while they remained fairly constant in hall exposure. Also, VS showed little, if any, increase for either group.

Decay. In decay, overall adaptation decreased with increasing time in the dark, $F(6,204)=23.17$, $p<.001$, but differentially as a function of different groups and tests, $F(12,408)=5.65, p<.001$. Figure 1 shows that only PS and NA for the handexposure group decreased with increasing decay time. The VS test in hand exposure and all three tests in hall exposure showed essentially no change over the decay period. Significance for the Tests by Groups interaction, $F(2,68)=23.19, p<.001$, again reflects the success of the hand vs. hall manipulation. With hand exposure, the average PS $\left(3.08^{\circ}\right)$ was greater than the average VS $\left(.58^{\circ}\right)$, while with hall exposure, the average VS $\left(1.54^{\circ}\right)$ was greater than the average PS $\left(-.42^{\circ}\right)$. Other significant sources of variance in decay were groups, $F(1,34)=31.19, p<.001$, the Tests by Time interaction, $F(12,408)=9.00, p<.001$, and the Groups by Time interaction, $F(6,204)=$ $14.89, \mathrm{p}<.001$.

Substantial decay of PS occurred following hand exposure, although decay was not complete. The $95 \%$ confidence limits for PS after $30 \mathrm{~min}$ in the dark do not include zero. In contrast, VS does not show consistent decay following hand exposure. These data constitute a reasonably accurate replication of those of Choe and Welch (1974). On the other hand, the hall exposure data did not replicate Redding (1975). VS did not decay following hall exposure. Therefore, the discrepancy noted in previous studies of VS decay cannot be due solely to differences in exposure conditions. However, the small amounts of VS obtained in the present study may have prevented detection of decay. Since the initial levels of VS obtained were small, any decay which could occur must also be small. In the limit, if no VS occurred, then no decay could occur. An adequate measurement of decay requires that substantial initial adaptation be obtained, which was clearly not the case in the present study.

Additivity tests. In hand exposure, the results are consistent with the two-component model. The sum of PS and VS measures is not statistically different from the NA measure when the data are averaged separately over exposure tests, $t(17)=1.33$, and decay tests, $t(17)=2.07$. However, the conclusion of additivity is qualified by the fact that there was very little VS. In acquisition, the VS test is significantly greater than zero only at the last exposure test, and the average VS $\left(-.04^{\circ}\right)$ is not different from zero $(95 \%$ confidence limits). In the decay period, while the average VS $\left(.58^{\circ}\right)$ is significantly greater than zero, three of the seven tests $(10,15$, and $25 \mathrm{~min}$ ) did not show significant change from zero. Thus, the additivity found may be due to the fact that no substantial VS occurred.

This conclusion is further supported by the observation that the average PS $\left(4.02^{\circ}\right.$ in acquisition and $3.08^{\circ}$ in decay) and NA $\left(3.02^{\circ}\right.$ in acquisition and $2.37^{\circ}$ in decay) were not significantly different from each other for either the exposure period, $F(1,68)=$ 3.12 , or the decay period, $F(1,68)=2.13$. It is reasonable to expect that if no VS occurs, twocomponent additivity will appear because NA and PS tests will measure the same adaptive component, namely proprioceptive adaptation. However, such data does not really test the two-component hypothesis since additivity could arise in such cases even if, in fact, the VS and PS components were not additive.

The fact that in hand exposure the NA measures are not numerically greater than PS measures suggests the further problem that the NA test may not be measuring the small amounts of VS that did occur. This suggestion is further emphasized by the results for hall exposure. Although still quite small, averaging $1.32^{\circ}$ in acquisition and $1.54^{\circ}$ in decay, VS was significantly greater than zero in all tests in both exposure and decay periods ( $95 \%$ confidence limits). On the other hand, none of the PS or NA tests were significantly different from each other. Clearly, the NA test is insensitive to the VS that occurred in hall exposure.

Again, as was the case for hand exposure, the sum of PS and VS tests is not statistically different from the NA test when the data are averaged separately over the exposure period, $t(17)=1.35$, and over the decay period, $t(17)=1.93$. However, such a comparison may be predicted solely on the basis of the small magnitude of the effects relative to error variance, and cannot be taken as evidence for the two-component, linear model.

\section{EXPERIMENT 2}

The first experiment was not immediately fruitful in answering our original questions. The largest problem is the small amount of VS, which precludes both an adequate test of the two-component hypothesis and accurate measurement of decay. Small VS on the order of $1^{\circ}-2^{\circ}$ is rather common in studies 
using variations on the Held \& Gottlieb (1958) apparatus during exposure (e.g., Choe \& Welch, 1974; Wallace, Note 1), however, much larger VS $\left(4^{\circ}-5^{\circ}\right)$ has been obtained when hallway exploration is used (e.g., Redding, 1973, 1975). The first experiment demonstrates that these differences in magnitude of VS cannot be entirely due to exposure differences, since VS is small in both hand and hall exposure, and this conclusion suggests the alternative possibility that the critical factor may be in the test procedures.

One possibility is that tests for VS conducted in a Held-Gottlieb box may suffer attenuation due to the subject's awareness of the limited range of target movement. The subject's knowledge of box size may produce a tendency to center the target within the box, which would reduce the measured VS (see also Harris, 1974; Wallach, Kravitz, \& Lindauer, 1963). This would seem particularly likely in the first experiment, where a lighted and clearly delimited field was present during test. In contrast, studies finding large VS (e.g., Redding, 1975) have not typically used test procedures which produce a limited frame of reference, either memorially or visually. Experiment 2 was designed to test this possibility.

The principal differences from the first experiment were that the test field was essentially unlimited and dark except for the target. Two groups were planned, 18 subjects each in hall and hand exposure, as in Experiment 1. However, after 12 subjects had been run in hand exposure, examination of the data revealed, contrary to expectation, larger VS than PS. The exposure apparatus differed from that of the first experiment in that the visual field was quite small. Consequently, the subject had to turn the eye to the right to view the optically displaced, but objectively straight-ahead, target. Thus, substantial VS could arise due to prolonged asymmetrical eye position (Ebenholtz, 1974; Paap \& Ebenholtz, Note 3). To control for such VS, an additional 12 subjects were run in hand exposure with a larger field of view and with the target objectively displaced to compensate for the optical displacement such that the target appeared straight ahead and an asymmetric eye position was not required to view the target.

\section{Method}

Subjects. The $\mathbf{4 2}$ male and female subjects were undergraduate volunteers at Illinois State University who received credit toward course performance for their participation.

Procedure. The procedure was similar to Experiment 1 except for the test apparatus and the hand exposure conditions. During hand exposure, the subjects traced, with the index finger and in a clockwise direction, a $3-\mathrm{cm}$ square viewed at eye level and arm's length (approximately $53.3 \mathrm{~cm}$ ) through a $20-\mathrm{D}$, base-left prism and in a plane parallel to the forehead. The first 12 subjects in hand exposure viewed the target square positioned objectively straight ahead, but perceptually displaced. A cardboard tube positioned between the wedge prism and target restricted the field of view to approximately $10^{\circ}$. For the second 12 subjects in hand exposure, the tube was removed, thereby increasing the field to approximately $20^{\circ}$, and the target was objectively positioned $11.4^{\circ}$ to the left such that it appeared straight ahead through the displacing prism. The procedure for hall exposure was the same as in Experiment 1, except that the subject wore a black cloak, reaching from neck to knee, as an additional precaution against their seeing any part of the body.

All tests were conducted on an open table in a room completely dark except for the test target. To test for VS, subjects verbally instructed the experimenter in adjusting a single luminous dot $(.25 \mathrm{~cm}$ diam) to appear straight ahead of the nose. For NA tests, the luminous dot was positioned objectively straight ahead, and the subject reached under a chest-high shield to point (sagittally) at the target. For PS tests, subjects simply pointed straight ahead under the shield with their eyes closed and with the field completely dark. The target dot was at eye level, and moved along an arc such that a constant distance of $121.9 \mathrm{~cm}$ from the subject was maintained. To minimize test time, while keeping equal practice on each test, only one starting position for the target dot was used in the VS tests: $8^{\circ}$ left of objective straight ahead. As in Experiment 1, all tests were conducted without prisms, and when vision was required, only the exposed, right eye was used.

Design. Subjects were alternately assigned to exposure conditions; however, after the first 12 subjects, the procedure was changed in hand exposure, and the last six subjects in hand exposure were run after data collection for the hall exposure was completed. Test order was counterbalanced and LA was defined as in the first experiment.

\section{Results and Discussion}

Separate analyses were performed on the data for the hand and hall groups, and within exposure conditions, acquisition and decay data were analyzed separately.

Hall exposure. Figure 2 shows results for the hall exposure. In acquisition, analysis of variance indicated significant effects for tests, $F(2,34)=20.62$, $p<.001$, and for exposure time, $F(4,68)=10.45$, $\mathrm{p}<.001$. The Test by Time interaction was not sig-

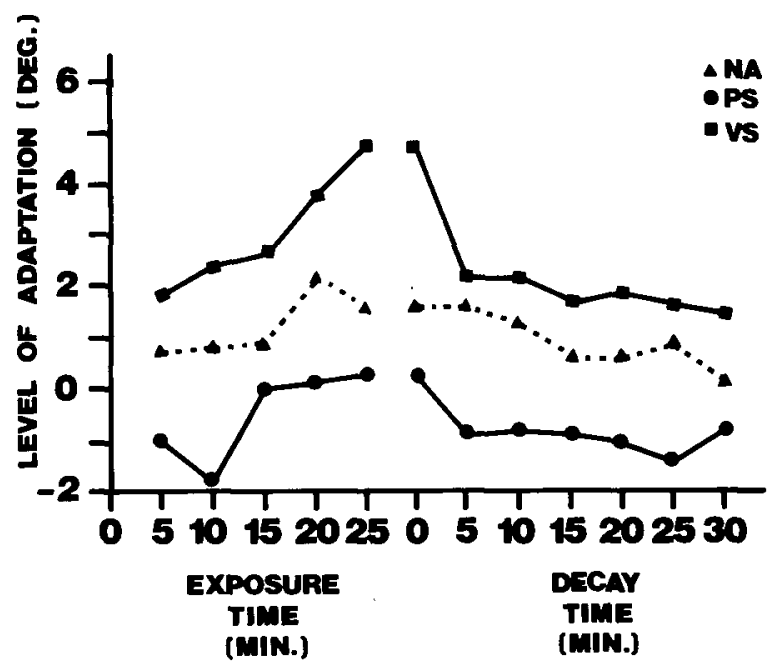

Figure 2. Level of adaptation as a function of exposure and decay time for hall exposure as measured by three kinds of tests: negative aftereffect (NA), proprioceptive shift (PS), and visual shift (VS). The positive direction indicates adaptive change for each test. Experiment 2. 
nificant, $F(8,136)=1.04$; however, the mean VS was significantly greater than zero ( $95 \%$ confidence limits) at all exposure times, while PS was never significant, and the NA measure showed significant effects only at the last two exposure tests ( 20 and $25 \mathrm{~min}$ ). Similarly, in decay, the only significant sources of variance were tests, $\mathrm{F}(2,34)=6.86, \mathrm{p}<.01$, and exposure time, $F(6,102)=5.44, p<.001$. However, PS was never significantly greater than zero, the NA decreased to zero by $15 \mathrm{~min}$ in the dark, and the $95 \%$ confidence limits for VS included zero after 20 min decay.

As predicted, VS increased substantially over that found in Experiment 1. This finding supports the hypothesis that subjects tend to center a target within the momentary or memorial visual field, and that this tendency acts to reduce VS effects in the Held-Gottlieb type of test apparatus. Even though VS was substantial, there is still little evidence of two-component additivity. In decay, the sum of PS and VS is not statistically different from the NA, $t(17)=.74$; however, this may be attributed to the small magnitude of effects. Moreover, in acquisition, the sum of VS and PS significantly exceeds (by $1.36^{\circ}$ ) the NA, $t(17)=2.40, p<.05$. As was the case in the first experiment, the NA test does not appear to be completely sensitive to VS.

The substantial decay of VS is similar to the findings of Redding (1975). Although decay was not numerically complete, VS is not statistically different from zero after $20 \mathrm{~min}$ in the dark, and there is a trend for the data to numerically approach zero. It seems reasonable, therefore, in light of previous findings of complete VS decay (i.e., Redding, 1975), to attribute the nonzero terminal LA in the present study to chance. More certainly, previous failures to find VS decay (i.e., Choe \& Welch, 1974) may be attributed to low initial adaptation. When large amounts of VS are obtained, clear evidence of decay appears, and such decay seems to be complete.

Hand exposure. Figure 3 shows results for hand exposure. Group 1 viewed the apparently displaced target with a narrow field of view, while Group 2 saw the apparently straight-ahead target in a wide field of view. Exposure time was a significant source of variance in both acquisition, $F(4,88)=9.94, p<.001$, and decay, $F(6,132)=10.31, p<.001$. The only other significant effect was the Tests by Groups interaction in acquisition, $F(2,44)=9.46, p<.001$, and in decay, $\mathrm{F}(2,44)=4.26, \mathrm{p}<.025$. This interaction reflects the fact that VS is greater than PS with the apparently displaced target (Group 1), while the converse is true when the target is apparently straight ahead (Group 2). In fact, the $95 \%$ confidence limits include zero for all PS data points in Group 1, and only the initial, nonadaptive effect for VS at $5 \mathrm{~min}$ is significantly different from zero for Group 2. The entirety of effects appears to be VS in Group 1 and PS in Group 2.

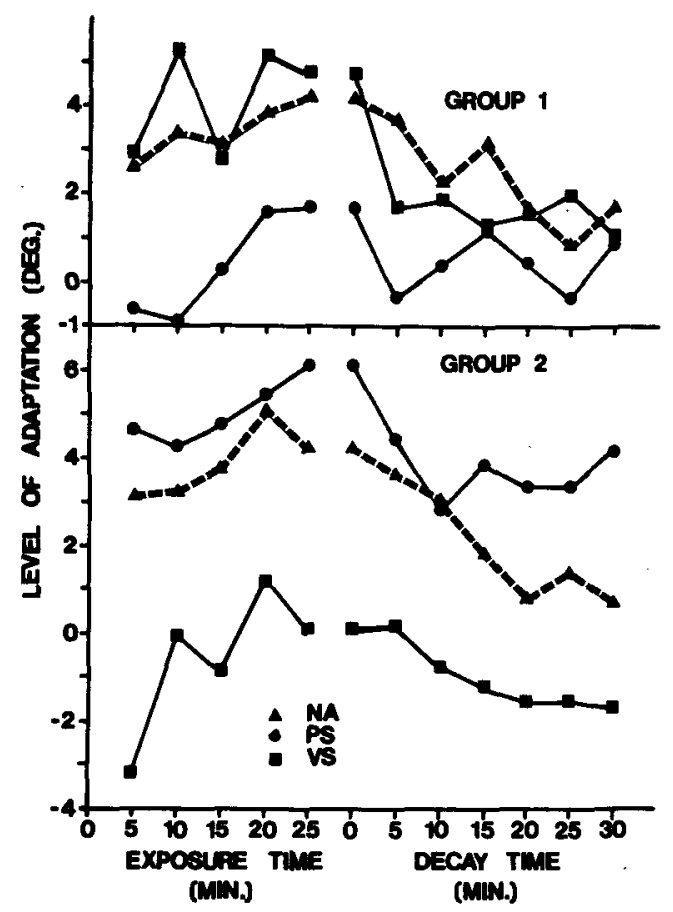

Figure 3. Level of adaptation as a function of exposure and decay time for hand exposure as measured by three kinds of tests: negative aftereffect (NA), proprioceptive shift (PS), and visual shift (VS). Data are shown separately for groups viewing apparently displaced exposure targets (Group 1) and apparently straight-ahead exposure targets (Group 2). The positive direction indicates adaptive change for each test. Experiment 2.

The similarity between VS in hall exposure (Figure 2) and in Group 1 of hand exposure suggests that VS might be entirely due to asymmetric target position during exposure. In hall exposure, unless subjects turn their trunk and/or head in the direction of the displacement, the eye maintains a more or less constant rotation in the head, Ebenholtz (1974; Ebenholtz \& Wolfson, 1975) has suggested that under such conditions VS can be attributed to eye-muscle potentiation, and Paap and Ebenholtz (Note 3) have marshalled an impressive array of parametric data arguing for the basic similarity between muscle potentiation effects and VS in adaptation studies. However, Craske (e.g., Craske \& Crawshaw, 1975) has argued for a true recalibration of eye position beyond eye-muscle potentiation effects. At least part of the VS in the present study can be attributed to muscle potentiation; however, the data do not allow a decision concerning whether all VS can be attributed to such effects.

The data for Group 2 constitutes a reasonably accurate replication of hand exposure in Experiment 1. Therefore, the differential effects of hand exposure on VS and PS may be attributed to exposure conditions, and do not appear to be due to any insensitivity of test procedures to VS. However, the data still do not provide much support for the two- 
component model. In Group 2, the sum of VS and PS is not statistically different from the NA in either acquisition, $\mathrm{t}(11)=.46$, or decay, $\mathrm{t}(11)=$ 1.06. Similarly, the value of VS + PS is not significantly different from the NA in Group 1 for either acquisition data, $t(11)=1.29$, or decay data, $t(11)=.18$. But such statistical, two-component additivity could arise simply because one of the two components is small in magnitude. This conclusion is particularly tenable in the decay data for Group 1, where the average NA $\left(2.52^{\circ}\right)$ is numerically close to the average VS $\left(2.05^{\circ}\right)$, as would be expected since PS is essentially zero $\left(.59^{\circ}\right)$. Moreover, there is a tendency in the data for both groups for the NA to be less than VS + PS, as was the case in Experiment 1 and in hall exposure of the present experiment. The consistency of this finding argues for its reality, and the direction of the effect is not easily accommodated by the two-component model.

The decay data for Group 1 supports the previous conclusion that VS decays completely when the amount of VS at the beginning of decay is sufficiently large to allow detection of the effect. The $95 \%$ confidence limits include zero for VS at 15, 20, and $30 \mathrm{~min}$. On the other hand, consistent with Experiment 1 , the data for Group 2 clearly shows that decay of PS is not complete. The confidence limits for PS never include zero for any of the decay tests. These findings suggest a basic difference in nature between visual and proprioceptive adaptation.

\section{CONCLUSION}

These experiments have clearly demonstrated the ability, via hand and hall exposure, to manipulate the relative magnitude of visual and proprioceptive shift. In addition, we have identified test procedures as a possible locus for differences in magnitude of VS, and we suggest that a tendency to center targets in the available test field is the important factor responsible for the small VS usually found with Held-Gottlieb test procedures. Moreover, the study has shown that, given a high LA, decay of VS is substantial, most probably complete. It seems reasonable to assume that previous failures to find VS decay can be attributed to low initial LA, making detection of decay problematical. On the other hand, the fact that PS decay is clearly not complete suggests a basic difference between the two kinds of adaptation.

Our efforts to test the two-component additivity hypothesis have been less rewarding. In a sense, the hand-hall manipulation is too successful, since in both exposure conditions the unemphasized component is virtually nonexistent, thereby making the statistical test ambiguous. The fact that one component increases when the other decreases suggests that VS and PS are causally and reciprocally related, as implied by the two-component model. However, it is also possible that such reciprocity arises from differential task demands which simply support one kind of adaptive change and not the other. Most of the exposure tasks used in adaptation can be characterized as hand or hall, but the present data indicates that such relatively gross manipulations are inadequate to test for relations between adaptive components. There is clearly a need to develop exposure tasks which produce substantial effects for both VS and PS.

Most puzzling and critical of the additivity hypothesis is the consistent finding that the sum of PS and VS numerically exceeds the NA. This effect is especially obvious in hall exposure, and significantly so in Experiment 2. There is a similar, but smaller and nonsignificant, tendency in hand exposure for both experiments. The reliability of this effect across variations in exposure and test conditions argues against its being a spurious result, and it is not subject to qualification by the small magnitude of one component. Previous two-component additivity failures (e.g., Templeton et al., 1974; Welch et al., 1974) have consisted of the NA exceeding PS + VS. In such cases, it has usually been possible to identify additional, but additive, components which make up the difference, however, this strategy is not possible for the present study. The NA test appears to actually be insensitive to a portion of the obtained change, particulariy to that measured by the VS test.

One rather obvious explanation for this effect has been considered, and at least tentatively rejected. Harris (1974) has suggested that much of the measured effects in adaptation experiments may be attributed to a "straight-ahead" shift in the egocentric direction which the subject treats as straight ahead, but without any true perceptual, proprioceptive or visual, recalibration of egocentric direction. For example, the apparent asymmetry of the subject in the environment when wearing displacing prisms may produce a cognitive shift of straight ahead in the direction of the displacement. Such a straightahead shift would lead to spurious "adaptation" on the VS test and "maladaptive" change on the PS test. The NA test, which requires pointing at a target without reference to straight ahead, would not be affected by such a straight-ahead shift. Therefore, VS should be greater than the NA, and PS should be in the negative direction. Such was the case with hall exposure in both experiments; however, Harris also proposes that the effects of straight-ahead shift should combine algebraically, in the same way as should true perceptual components. The negative contribution of straight-ahead shift to the PS test should cancel the positive contribution to the VS test, therefore producing additivity. This prediction was clearly not met in the present experiments. Moreover, in hand exposure a straight-ahead shift would predict a NA greater than PS since PS would be attenuated 
and not the NA. Again, prediction based on a straight-ahead shift was not upheld.

Harris may be incorrect in assuming that a straightahead shift does not affect the NA test, especially when PS is also assessed in the same experimental paradigm and when a single, straight-ahead NA target is used. The subject may form an association between the straight-ahead position in space and the position of the NA test target, assuming them to be the same. Thus, if the subject tends to point "straight-ahead" rather than at the target per se in the NA test, magnitude of the NA would be reduced, leading to the result PS + VS $>$ NA. A test of this hypothesis would require multiple positions for the NA target to prevent the formation of such an association.

It may, therefore, be possible to arrive at an explanation for the present data in terms of present theory and known mechanisms of change; however, we feel that a final resolution of the additivity question awaits development of a clear conceptual alternative to the linear model. Such theoretical development will permit more powerful, direct tests between additive and nonadditive hypotheses.

\section{REFERENCE NOTES}

1. Wallace, B. Effects of targets and exposure time on magnitude of adaptation to prisms. Unpublished manuscript, 1975. (Available from the author.)

2. Redding, G. M. Visual and proprioceptive adaptation to optical tilt. Paper presented at the Midwestern Psychological Association meetings, Chicago, Illinois, May 1975.

3. Paap, K. R., \& Ebenholtz, S. M. Perceptual consequences of post-tetanic-potentiation: An alternative explanation for adaptation to wedge prisms. Paper presented at the Psychonomic Society meetings, Denver, Colorado, November 1975.

\section{REFERENCES}

Chое, C., \& Welch, R. B. Variables affecting the intermanual transfer and decay of prism adaptation. Journal of Experimental Psychology, 1974, 102, 1076-1084.

Craske, B., \& CRAWShaW, M. Oculomotor adaptation to prisms is not simply a muscle potentiation effect. Perception \& Psychophysics, 1975, 18, 105-106.

EBEnholtz, S. M. The possible role of eye-muscle potentiation in several forms of prism adaptation. Perception, 1974, 3, 477-485.

Ebenholiz, S. M., \& Wolfson, D. M. Perceptual after-effects of sustained convergence. Perception \& Psychophysics, 1975, 17, 485-491.
Hardt, M. E., Held, R., \& Steinbach, M. J. Adaptation to displaced vision: A change in the central control of sensorimotor coordination. Joumal of Experimental Psychology, 1971, 89, 229.239.

HARRIs, C. S. Beware the straight-ahead shift: A nonperceptual change in experiments on adaptation to displaced vision. Perception, 1974, 3, 461-476.

HAY, J. C., \& PICK, H. L., JR. Visual and proprioceptive adaptation to optical displacement of the visual stimulus. Journal of Experimental Psychology, 1966, 72, 419-444.

HELD, R., \& Gortures, N. Technique for studying adaptation to disarranged hand-eye coordination. Perceptual and Motor Skills, $1958,8,83-86$.

Melamed, L. E., Halay, M., \& Gildow, J. W. Effect of external target presence on visual adaptation with active and passive movement. Journal of Experimental Psychology, 1973, $98,125-130$.

McLaughlin, S. C., \& Webster, R. G. Changes in straightahead eye position during adaptation to wedge prisms. Perception \& Psychophysics, 1967, 2, 37.44.

Redding, G. M. Visual adaptation to tilt and displacement: Same or different processes? Perception \& Psychophysics, 1973, 14, 193-200.

Redoing, G. M. Decay of visual adaptation to tilt and displacement. Perception \& Psychophysics, 1975, 17, 203-208.

Templeton, W. B., Howard, I. P., \& Wilkinson, D. A. Additivity of components of prismatic adaptation. Perception \& Psychophysics, 1974, 15, 249-257.

W ALLACE, B. Target and arm observation effects on adaptation to prism displacement. Perception \& Psychophysics, 1974, 15, 145-148.

Wallace. B., \& Garrett, J. B. Perceptual adaptation with selective reduction of felt sensation. Perception, 1975, 4, 437.445.

Waleach, H., Kravitz, J. H., \& Lindauer, J. A passive condition for rapid adaptation to displaced visual direction. American Journal of Psychology, 1963, 76, 568-578.

Welch, R. B., ChoE, C. S., \& Heinrich, D. R. Evidence for a three-component model of prism adaptation. Journal of Experimental Psychology, 1974, 103, 700-705.

Wilkinson, D. A. Visual-motor control loop: A linear system? Journal of Experimental Psychology, 1971, 89, 250-257.

\section{NOTE}

1. Lateral target pointing differs from the frequently used sagittal target pointing; however, Wallace (1974) found essentially the same performance when the two types of movement were made rapidly. Therefore, at least for the eye-hand coordination test (i.e., the NA test), there is no reason to believe that the present procedure produces performance different from studies using sagittal pointing.

(Received for publication July 12, 1976; revision received September 20, 1976.) 